\title{
ORACIONES DE RELATIVO Y VARIACIÓN MODAL EN EL HABLA CULTA COSTARRICENSE
}

\author{
JORGE MURILLO \\ Universidad de Costa Rica
}

La selección modal en las oraciones relativas ha sido bastante estudiada en los últimos años ${ }^{1}$. No obstante, a pesar de los numerosos asedios hechos desde diferentes marcos metodológicos, no se ha logrado aún esclarecer cuáles son los mecanismos que actúan como rasgos seleccionadores del modo en este tipo de oraciones.

A continuación, se hace un análisis de la elección del modo en las oraciones adjetivas, circunscrito específicamente al habla culta costarricense y a construcciones en presente. El estudio se enmarca dentro del Proyecto de estudio coordinado de la norma lingüística culta de las principales ciudades de Iberoamérica y de la Península Ibérica. No obstante, no responde solamente a esos lineamientos sino que también incorpora otros criterios (no especificados en el cuestionario original) para lograr una visión de conjunto en el análisis y la interpretación de los datos.

Se trabajó con 36 entrevistas ( 20 horas de grabación, con 32 entrevistas de media hora cada una y 4 de 45 minutos) en las cuales participan 46 informantes, divididos de la siguiente forma: 10 informantes de la I generación (de entre 25 y 35 años, que correspoden al 21,75\%); 21 de la II generación (de entre 36 y 55 años, para un total de 45,65\%) y 15 de la ter-

${ }^{1}$ Guitart, «The NP-Based Analysis of Mood Choice in Spanish Relative Clauses», Studies in language learning and Spanish linguistics. In honor Tracey Terrel. Editado por P. Hashemipour, R. Maldonado y M. van Naerssen, New York, McGraw-Hill Inc., 1994. págs. 385398 , da una de las últimas interpretaciones del fenómeno de la variación modal en las oraciones adjetivas, el cual ya había sido abordado por los estudios de gramática tradicional (RAE, Esbozo de una nueva gramática de la lengua española. Madrid, Espasa Calpe, 1973) o los enfoques semántico-generativos (Rivero, Estudios de gramática generativa del español. Madrid, Cátedra, 1979, por ejemplo). Los anteriores solo para mencionar algunos estudios que se centran específicamente en este tema; existen, de hecho, numerosas referencias en todos los manuales de gramática del español o en análisis sobre el sistema verbal del español.

RFE, LXXX, 2000, $1 .^{\circ}-2 .^{\circ}$, págs. 141-153 
cera (mayores de 55 años, un 32,60\%). Del total de informantes, $50 \%$ son mujeres y $50 \%$, hombres ${ }^{2}$.

Los informantes son de la zona metropolitana (San José), por cuanto ésta es el foco de irradiación cultural del país. Todos los informantes tienen educación superior; en su mayoría son egresados o estaban en los últimos años de estudio. Se seleccionaron atendiendo a los siguientes factores socioculturales: ambiente familiar, grado de instrucción (incluyendo lecturas, viajes y dominio de lenguas extranjeras), ocupación. Además, se consideró que fueran nacidos en el área metropolitana o al menos que hubieran residido en esta zona durante tres cuartas partes de su vida. Debían ser hijos de hispanohablantes, preferentemente nacidos en la misma ciudad y también debían haber recibido toda su instrucción formal -incluyendo la superioren la misma ciudad.

El grupo de relativas que analizamos corresponde a aquellas en las que es factible la variación modal. El criterio que se siguió para determinar lo anterior fue la simple permutación de la forma verbal: si aceptaba ambas formas, no importando con cuál apareciera en el corpus, se clasificó como susceptible de variación.

Según el modo que usan, tenemos las siguientes frecuencias de aparición: con subjuntivo, 60 casos $(61,86 \%)$ y con indicativo, 37 casos $(38,14 \%)$.

Todas estas oraciones tienen antecedente [-específico] y, por lo tanto, deberían aparecer con forma verbal subjuntiva, según los principios tanto de la gramática tradicional como de las perspectivas modernas ${ }^{3}$. Veamos dos casos del corpus, tomados al azar, para ejemplificar lo afirmado:

(1) Habrá otras... otros portillos que tal vez más de una gente aprovecha.

(2) Es que depende de la situación que te vengan a plantear.

En (1), el sustantivo portillos (oportunidades, en el habla costarricense) es totalmente inespecífico y la relativa que lo acompaña debería estar en

\footnotetext{
${ }^{2}$ Los datos fueron recolectados por la Dra. Yamileth Solano, catedrática de la Universidad de Costa Rica. La recolección la llevó a cabo a finales de los ochenta como parte de su investigación doctoral sobre los conectores pragmáticos en el habla culta costarricense, bajo la dirección del Dr. Lope Blanch.

${ }^{3}$ Por ejemplo, para la RAE, Esbozo de una nueva gramática de la lengua española, Madrid, Espasa Calpe, 1973, pág. 456, posición representativa de los estudios de corte tradicional, el critero de selección se resume de la siguiente forma: «En las oraciones de relativo, el verbo va en indicativo cuando el antecedente es conocido; si el antecedente es desconocido o dudoso, el verbo subordinado se pone en subjuntivo». Dentro de los enfoques generativos, se parte también de este mismo criterio al cual se agregan algunos matices dependiendo de la opinión del investigador: Cf. Rivero, op. cit.; Hadlich, Gramática transformativa del español, Madrid, Gredos, 1982; Bull, Spanish for Teachers. Applied Linguistics, New York, The Ronald Press Co., 1965, y ya con una nueva visión, Guitart, art. cit.
} 
subjuntivo (Habrá otros portillos que más de una gente aproveche). En (2), la situación a que se refiere el hablante es inespecífica también, pero ahí sí aparece el subjuntivo, aun cuando bien podría haber dicho: Es que depende de la situación que te vienen a plantear, con indicativo, sin variar el carácter semántico de [-especificidad] del sintagma antecedente.

La conclusión que salta a la vista, en primer lugar, es que el rasgo de [-especificidad] de los sintagmas no necesariamente está marcado por el subjuntivo, pues en su lugar podría aparecer el indicativo, sin perjuicio del sentido o de la comprensión de los enunciados ${ }^{4}$. La segunda conclusión, derivada de la anterior, es que el subjuntivo se está convirtiendo en un rasgo redundante de marcación de la [-especificidad] en las oraciones de relativo en vista de que otros elementos (que analizamos en detalle adelante) asumen su papel, dejándolo en la casilla de opcional. Esta segunda conclusión tiene como corolario el que la oposición modal indicativo/subjuntivo se haya neutralizado en contextos donde la [-especificidad] está marcada por otros elementos, como se puede apreciar en (3):

(3) Porque eh... que una persona, digamos, llegue y me diga una vulgaridad en cierto medio puede faltarme al respeto dependiendo de las personas que me rodean en ese momento y de la confianza que haya.

en donde aparecen dos oraciones relativas coordinadas en distinto modo, aunque ambas se refieren a antecedentes [-específicos].

Por otra parte, si tomamos en cuenta los porcentajes de aparición del indicativo en estos contextos, restringidos normalmente al subjuntivo, podemos apreciar en qué medida este último está perdiendo terreno. Esta reducción en los dominios del subjuntivo se vería aún más acentuada si se considera que, hipotéticamente, los 97 enunciados serían susceptibles de aparecer en indicativo. No obstante, partiendo de los datos obtenidos tenemos

\footnotetext{
${ }^{4}$ Ross y Umaña, «La desaparición del subjuntivo español y sus implicaciones para el cambio lingüístico», Revista de Filología y Lingüística, XVII, 1-2, 1991, págs 193-202, analizan las consecuencias semánticas del uso indistinto del indicativo o subjuntivo con este tipo de oraciones. Toman, como ejemplo, el enunciado El que llega primero a la meta gana, que se puede interpretar como -específico (en ese caso requeriría subjuntivo) y como [+específico], con indicativo, y apuntan que, con antecedente [-específico] pero con indicativo, la oración no induciría a ningún hablante a considerarla como ambigua; es decir, con cualquiera de los dos modos verbales, los hablantes costarricenses la interpretan como -específica. Por eso, afirman: «Pero la verdad es que aunque la oración es ambigua, las consecuencias de entender el sentido contrario al que el hablante tenía en mente son prácticamente nulas. De hecho, es probable que muchas personas no captarían sin hacer un esfuerzo la diferencia entre ambos sentidos. Puesto que la tendencia general es hacia la sustitución del subjuntivo por el indicativo, y puesto que en ejemplos como éste la diferencia entre ambos modos es absolutamente mínima, se puede usar el indicativo en todos los casos sin temor a repercusiones negativas» (pág. 220).
} 
que, por lo menos para el habla culta costarricense, el subjuntivo, aunque opcional, sigue manteniendo su hegemonía cuando el hablante debe elegir entre los dos modos que se le ofrecen, al contrario de lo que creen Ross y Umaña, quienes afirman que «se observa una notoria disminución del uso del subjuntivo en este entorno» 5 .

Pasemos a analizar los enunciados para tratar de encontrar cuáles son los factores que potencian la variación modal y qué otros mecanismos está utilizando el habla culta costarricense para marcar los sintagmas [-específicos]. Con ello, se pueden determinar las causas del debilitamiento de las funciones tradicionales del subjuntivo dentro del sistema verbal.

En primer lugar, tenemos un reducido número de casos (4) en los que aparece un cuantificador en función adjetiva dentro del sintagma nominal antecedente:

(4) Cualquier persona que vaya a trabajar...

(5) Entonces, cualquier aumentillo que te puedan haber hecho en el año, ¿dónde se te va?

(6) No, el maestro puede trabajar también en otra área que no sea oficial.

Aquí, el elemento que pareciera marcar el rasgo de [-especificidad] es el cuantificador, en tanto es posible usar el indicativo en las relativas sin que haya variación de sentido. De hecho, parece existir una atracción modal entre los cuantificadores de negación o indeterminación existencial y el subjuntivo, dado que no se documentaron entornos similares donde se usara el indicativo. Sin embargo, no se podría justificar el carácter [-específico] del antecedente solarnente acudiendo al uso del cuantificador, toda vez que bien podría haber aparecido un artículo (definido o indefinido) y la variación modal también hubiera sido posible. Con lo anterior se quiere destacar el hecho de que también la intención del hablante o su experiencia previa juegan un papel pragmático fundamental para la marcación del antecedente $\mathrm{y}$, por lo tanto, para la elección optativa del modo verbal. Tomemos, por ejemplo, el caso (5) y sustituyamos el cuantificador por artículos: Entonces \{cualquier/ el/ un\} aumentillo que te \{puedan/pueden\} haber hecho en el año, ¿dónde se te va? Obviamente, todas las combinaciones anotadas son posibles con significado de [-especificidad], lo que nos lleva directamente a la pregunta: ¿cómo se está marcando entonces la no especificidad del antecedente? y, más importante para nuestro punto de vista, ¿por qué se ha desplazado al subjuntivo de esa función? Las respuestas a estas preguntas solo es posible darlas en tanto echemos mano a otros recursos, de índole extra-

\footnotetext{
${ }^{5}$ Op. cit., pág. 193.
} 
lingüística, y por eso a veces ignorados por los estudios que se ciñen dentro de marcos puramente lingüísticos. Si un hablante, por ejemplo, escucha la frase El aumentillo que te pueden haber hecho en el año, ¿dónde se te $v a$ ?, no tendría duda alguna de que el aumentillo es un sintagma no específico, aun cuando no se use cuantificador o el modo subjuntivo. La no especificidad, entonces, viene dada por el contexto de comunicación y por los presupuestos del proceso de comunicación que en ese momento se lleva a cabo. Si bien es cierto que ciertos elementos sintácticos (uso de cuantificadores indefinidos, uso de verbos modales), morfológicos (diminutivos) o pragmáticos (la pregunta al final del período) ayudan a conseguir el efecto de [-especificidad] en el sintagma y, por consiguiente, a privar al subjuntivo de su función, no es menos cierto que la intención del hablante y el entorno comunicativo son parte esencial de este proceso.

No obstante lo anterior, se pudieron detectar algunos procedimientos que parecen incidir, de forma directa, en la marcación de la [-especificidad] del antecedente, de los cuales damos cuenta en los párrafos siguientes. Antes, sin embargo, deben hacerse dos observaciones preliminares a) aunque el subjuntivo haya visto restringida su función exclusiva de marcación de la [-especificidad], aún así puede desempeñar esa función en casos en que no haya otros sustitutos y b) las afirmaciones se hacen específicamente para los datos aportados por las encuestas y, por ello, de ninguna manera son extensivas (aunque pudieran serlo) a la lengua española en general.

Además, insistimos en que los casos de relativas con variación modal responden a criterios muchas veces híbridos y otras, a cirscunstancias particulares de situación comunicativa. Eso es de esperar en un área sintáctica y semántica de la lengua que, según nuestro criterio, está en vías de reorganización $\mathrm{y}$, por consiguiente, muestra espacios de inestabilidad e indefinición. Por ello, lo que aquí se proponen son lineamientos generales que nos permitirán, más que encasillar los enunciados en tendencias específicas, dar cuenta del rumbo que están tomando los cambios dentro del ámbito de las oraciones relativas y la selección modal.

En primer lugar, hay casos en que se da una clara marcación de la [-especificidad] del antecedente por medio de recursos léxico-semánticos, es decir, que aparecen en la oración subordinante clases de palabras que dan al antecedente ese carácter. El grupo más importante lo constituyen las nociones asociadas con [+/-dependencia], expresada tanto en forma verbal como adverbial (10 casos):

(7) es que depende de la situación que te vengan a plantear.

(8) ...la muerte o lo que tiene que pasar muchas veces en la vida pasa, independientemente de lo que uno haga voluntariamente para que pase o no en ese momento. 
(9) $\mathrm{Y}$ que en el fondo la vida siempre sigue adelante, independientemente de lo que uno piensa, diga o haga.

(10) Pero eso va condicionado, digamos, al momento y lugar en que yo esté y dependiendo de la confianza, digamos, que haya en el grupo.

Se aprecia claramente cómo todo aquello que se incluya en el campo semántico de la [+/-dependencia] tipifica el antecedente de la relativa como [-específico]. La redundancia del subjuntivo para marcar ese carácter se puede ver tanto en (3) como en (9), pues en ambos casos una de las oraciones relativas aparece en indicativo, alternando con otra(s) en subjuntivo ${ }^{6}$. En algunos enunciados, como en (10), la marcación va reforzada por otras clases de palabras que manifiestan el carácter [-específico] del antecedente: va condicionado y digamos, por ejemplo. Salvador Fernández $\mathrm{z}^{7}$ denomina este tipo como subjuntivo de indeterminación:

Hay especialmente un subjuntivo de INDETERMINACión cuando el antecedente va precedido de ségún, depende de y verbos análagos (...). El nombre en singular es con mucha frecuencia un concepto abstracto: acción, cualidad, estructura, idea, acto psíquico, etc. El subjuntivo pone de relieve la indeterminación cualitativa, gradual, estructural con que es pensado el objeto.

En otro subgrupo, tenemos cuatro enunciados con la siguiente estructura SER $+\{$ RAR- $\}+$ RELATIVA (neg), en la que el adjetivo califica al antecedente, pero no especificándolo:

(11) ...por ejemplo, el cine son treinta colones de entrada y después, por lo general, de la salida del cine es rara la persona que no pase a alguna soda a... algo.

(12) Lo reconozco de que ahí sí, por ejemplo, es rara la persona que no vaya a misa.

El sentido altamente valorativo y subjetivo que imprime la noción semántica de rareza a las frases, es suficiente para designar al antecende como [-específico].

Los adverbios también pueden indicar el carácter [-específico] del antecedente

(13) Yo dificilmente he encontrado un chiquito que le gusten los payasos. (14) ...porque él es un verdadero pionero en la investigación científica, verdad, no solo un gran científico sino el que pone las bases para lo que luego se pueda hacer.

\footnotetext{
${ }^{6}$ La alternancia modal en esta frase puede explicarse también por factores de atracción morfológica por proximidad o parecido de las desinencias: piensa, aunque es indicativo, tiene el mismo morfema de las formas diga y haga, subjuntivas.

${ }^{7}$ Salvador Fernández Ramírez, Gramática española. 4. El verbo y la oración, editada por Ignacio Bosque, Madrid, Arco Libros S.A., 1986, pág. 379.
} 
Como se aprecia, la modalización en (13) por medio del adverbio supone que el antecedente no es específico y la marcación de hecho futuro en (14), y por lo tanto incierto, la da el adverbio luego.

Otros elementos morfológicos, sintácticos y pragmáticos también colaboran con el subjuntivo en la marcación de la [-especificidad]. Enumeramos algunos ejemplos de entre los casos restantes para que se pueda apreciar la variedad existente:

a) Futuro o condicional simples de indicativo en la cláusula principal:

(15) No, pero se... se... se entiende... el costo que signifique saldrá del... de las... de las ventajas económicas del proyecto.

(16) Yo diría que el respeto va aparejado con... con el conocimiento que tengamos nosotros de cada persona.

b) Verbos de opinión o valoración en la cláusula principal [Bustos ${ }^{8}$ los denomina operadores epistémicos de creencia], por ejemplo, pensar y creer:

(17) Yo creo que el respeto va identificado directamente con... con... con la forma o con el entendimiento que yo tenga del amor.

(18) Y además de que yo pienso que el pensionado que se retire totalmente de la actividad se enferma.

Bien podría argumentarse que si se elimina el verbo principal, la cláusula subordinada mantendría su doble capacidad de elección modal, pero debe notarse cómo el hablante claramente supedita todo su enunciado al carácter semántico del verbo principal $\mathrm{y}$, por ende, modaliza todo el período sintáctico haciéndolo aparecer como una mera opinión, no como un hecho verdadero o específico. En términos de la modalidad, pareciera que el enunciado es declarativo, pero en el fondo lo que predomina es la modalidad epistémica.

Como contraparte, la modalidad deóntica también influye en la caracterización de la inespecificidad, pues le imprime al enunciado un carácter generalizador, pensado como necesario por el hablante y por lo tanto futuro. Las perífrasis obligativas cumplen esa función en los siguientes casos:

(19) Pero sumados a esos factores ya ancestrales en el ser costarricense hay que añadirle nuevos factores que puedan servir de catalizadores culturales...

(20) Entonces para eso hay que hacer un estudio propio de las especies de la región y que estén situadas en zonas biológicas.

Desde la perspectiva del hablante, la obligación expresada en presente se concibe siempre con carácter futuro, lo cual va reforzado, por ejemplo

\footnotetext{
${ }^{8}$ Pragmática del español: negación, cuantificación y modo, Madrid, UNED, 1986.
} 
en (19), con el adjetivo nuevos y con el tono de finalidad que adquiere (20) por medio del sintagma para eso.

c) Contexto sintáctico de aparición: en estos casos, la relativa aparece incrustada dentro de otra construcción que rige subjuntivo, lo que produce una suerte de consecutio temporum o asimilación modal:

(21) Es posible que ese sea un proceso que se pueda dar también en el adulto, si el pensamiento es demasiado rápido.

(22) No es que él, por ejemplo, si son vacas que sea una vaca realista que usted le sienta la piel, el músculo, todo.

(23) Para mí, eso de que lleven un curso que les enseñe cómo van a hacerse el nudo de la corbata... todas esas cosas, me parece que es un desperdicio que pasen un semestre aprendiendo esas cosas.

(24) Ojalá todos los que salgan a hacer estudios fuera de Costa Rica tomen ese ejemplo...

En todos los casos anteriores, la relativa aparece incrustada en una construcción sintáctica más compleja, que a su vez requiere de subjuntivo. Lo anterior ya lo había observado Gonzalo ${ }^{9}$ al afirmar:

$\mathrm{Si}$ ampliamos nuestras consideraciones al contexto oracional parece que el verbo principal, como eje de la oración, ha de actuar sobre la mención del SN en aquellos casos en que no viene determinada desde adentro, en unión de factores como la función de sujeto u objeto de la relativa, la correlación de tiempos entre el verbo principal y el subordinado y el significado mismo del verbo principal.

d) Relativos de generalización: en estos enunciados, la relativa hace mención genérica aludiendo a todos los elementos de una clase, lo que de por sí entrañaría una ausencia de especificación:

(25) Toda actividad humana que se precie de mejoramiento debe estar en crisis, de lo contrario nunca mejoraríamos.

(26) El que se agüeve (aburra) pierde.

(27) Bueno, mirá, podés hablar todo lo que te dé la gana, aquí me espero pacientemente hasta que terminés de hablar...

Para Fernández Ramírez ${ }^{10}$, «...el antecedente del relativo hace en general la referencia al objeto o a los objetos con una intención nueva, intención que el subjuntivo trata de potenciar». Desde esta perspectiva, todos estos tipos de ora-

\footnotetext{
${ }^{9}$ Carmen R. Gonzalo, «La alternancia modal en las relativas y los tipos de mención del SN complejo», Indicativo y Subjuntivo, compilado por Ignacio Bosque, Madrid, Taurus Universitaria, 1990, pág. 296.

${ }^{10}$ Op. cit., pág. 384 .
} 
ciones usarían el subjuntivo, pero se ha podido comprobar con el recuento hecho para este corpus que eso no es así. La relativa de generalización es susceptible de aceptar tanto el indicativo como el subjuntivo (se contabilizaron 31 oraciones de relativo de generalización, de las cuales 16 aparecieron con subjuntivo y 15 con indicativo, siendo todas susceptibles de aceptar cualesquiera de los dos modos). Esto demuestra que la sola mención generalizada del antecedente se basta para marcar la no especificidad, dejando la casilla verbal de la relativa como opcional en cuanto a selección modal se refiere.

Los anteriores constituyen los mecanismos más comunes, encontrados en el corpus, que colaboran con la mención del antecedente y, a la vez, en la mayoría de los casos hacen innecesaria la aparición obligada del subjuntivo. Los casos que ameritaban una explicación pormenorizada y que eran únicos, no se incluyeron en vista de su carácter aislado.

En cuanto a las oraciones relativas que se suponía, de acuerdo con la gramática tradicional, debían aparecer con subjuntivo pero en su lugar usan el indicativo, la gran mayoría quedan incluidas dentro de los lineamientos generales esbozados hasta aquí.

De particular interés dentro de este segundo subgrupo resultan aquellos períodos donde aparece un adverbio de duda (entornos tradicionalmente marcados como típicos para la aparición del subjuntivo), pues todos ellos escogieron el indicativo:

(28) Que posiblemente hay cosas que están ahí de veras, ¿verdad?

(29) Son concepciones que tal vez son tradicionales, ¿verdad?

En los entornos anteriores el hecho de que el hablante (como se demostró en el capítulo sobre el subjuntivo en construcciones independientes) prefiera el indicativo para la expresión de la duda, influye sobremanera en la selección modal de la relativa, máxime si se toma en cuenta que el verbo principal del periodo también aparece en indicativo.

Como observación general, se puede plantear que en la mayoría de casos en que las relativas, aun con antecedente no específico, aparecieron con indicativo, priva un sentimiento del hablante de que lo mencionado se acerca más a lo específico, sea por intuición de una referencia real o por conocimiento previo:

(30) La idea es darle a los estudiantes que van a ser maestros elementos para que se puedan defender como tales.

(31) Y digo en principio 'para uno mismo', porque quien no puede respetarse a sí mismo no puede ser respetado por los demás.

(32) Yo creo que el padre debe estar consciente de las dificultades del hijo, debe colaborar con él, eh... como una guía, como un punto de apoyo, pero no como una persona que le hace la tarea. 
En los tres ejemplos anteriores, el hablante está haciendo referencia a antecedentes no especificados dentro del contexto comunicativo, pero prefiere usar el indicativo porque, lo más problable, existe un conocimiento previo (del mundo real) que le permite hacer referencia a esos antecedentes como si hubieran existido (porque los conoce, los ha visto o tiene la firme convicción de que existen). No se puede argumentar que esos antecedentes sean específicos, ya que no lo son, pero sí se puede asegurar que dentro de la experiencia de esos sujetos, se los concibe como muy cercanos a su realidad.

A este respecto, Ross y Umaña ${ }^{11}$ se habían planteado la hipótesis siguiente:

En vez de existir simplemente dos rasgos de especificación ([+especifico] $y$ [-especifico]), hay un continuum entre ambos extremos. Cuando mayor sea el grado de no especificidad, tanto mayor la probabilidad de que se emplee el subjuntivo.

la cual no llegan a comprobar pues aducen «que había tanta variación de un hablante a otro, que nos resultó difícil identificar un patrón claro a lo largo del presunto continuum de especificidad» ${ }^{12}$. Interesante resulta la idea de estos lingüistas en tanto se desliga del trillado binarismo en el que se pretende encasillar las variedades lingüísticas y, además, supone que ciertas observaciones (aunque empíricas) los llevaron a plantear la existencia de ese continuum.

Una comprobación de la hipótesis anterior necesitaría un estudio diseñado para tal efecto, pero en nuestro caso, en que manejamos una buena cantidad de datos, se puede apreciar cómo el uso del subjuntivo en las relativas se vuelve menos obligatorio tanto más se acerque el hablante a la consideración de un antecedente muy cercano a lo específico. El establecimiento de los puntos exactos en donde ese continuum pasaría de indicativo a subjuntivo es muy difícil de marcar con seguridad en vista de que, como hemos visto, hay elementos externos a lo propiamente lingüístico que entran en juego en la selección modal y por razón de que (quizá lo más importante) la inestabilidad de esta parcela del sistema verbal no permitiría, por ahora, establecer patrones totalmente definidos.

Finalmente, vale la pena hacer algunas observaciones sobre los factores pragmáticos involucrados en la selección del modo de las relativas. Desde esta perspectiva se trata de explicar la escogencia de uno $u$ otro modo tomando en cuenta las repercusiones que esa elección pueda tener dentro del contexto comunicativo. Así, se parte de la idea (Rivero, por ejemplo13) de

${ }^{11}$ Op. cit., pág. 194.

12 Ibidem, pág. 197. 
que, si el hablante escoge el indicativo en una relativa, tiene la convicción de que el sintagma nominal tiene una referencia específica y cuando usa el subjuntivo, alude a un antecedente no específico. El problema se presenta cuando una misma oración, que hace referencia a un antecedente [-específico], puede elegir indistintamente cualesquiera de los modos, sin por ello haya repercusiones significativas en la semántica del enunciado, tal y como se ha visto a través de nuestro análisis y como lo comprueban Ross y Umaña ${ }^{14}$.

Lo anterior nos conduce a concluir, con Umaña y Ross, que la especificidad del antecedente no es un factor decisivo en la escogencia modal y que ésta parece estar más bien marcada por otros elementos de índole diversa. De esta manera, el uso del subjuntivo o el indicativo sigue siendo un problema sin resolución definitiva.

Bustos $^{15}$, considera el asunto haciendo uso de los conceptos de implicatura, al plantear que «cuando la oración relativa está en indicativo es causa de una implicatura convencional, mientras que cuando está en subjuntivo es origen de una implicatura conversacional». Por ejemplo, en el enunciado Los que llegan primero recibirán un premio, el hablante usa convencionalmente los modos (indicativo y subjuntivo), es decir, que la relación no es cancelable (Hay algunos que en verdad llegan y que reciben los premios). Si el mismo enunciado emplea el subjuntivo Los que lleguen primero recibirán los premios, la implicación es de carácter conversacional y es cancelable (es decir, yo no estoy seguro de que alguien reciba el premio porque quizá ninguno vaya a llegar a la meta). Con el indicativo, el sujeto emite un enunciado en cuya verdad cree por experiencias anteriores que ha tenido; con el subjuntivo, el sujeto se mantiene neutral ante el valor veritativo de su enunciado y, precisamente por ello, puede cancelarlo o negarlo con otro de carácter opuesto.

Esta solución podría plantearse para explicar aquellos casos en los que no existe, aparentemente, ningún otro factor que influya en la selección modal. Por ejemplo, en

(33) En una situación así hay quien diga: «lo que ha de pasar, pasará».

asalta la duda de por qué el hablante usa distintos modos verbales en las relativas si evidentemente todo el enunciado hace referencia a una situación no específica (lo sabemos por el contexto). La explicación, de acuerdo con

13 «Especificidad y existencia», Indicativo y Subjuntivo, compilado por Ignacio Bosque, Madrid, Taurus Universitaria, 1990, págs. 261-279.

${ }^{14} \mathrm{Op}$. cit.

15 Op. cit., pág. 213. 
el enfoque de Bustos ${ }^{16}$, sería la siguiente: cuando enuncia quien diga usa el subjuntivo porque no está seguro del valor veritativo de su enunciado (quizá nadie vaya a decir nunca eso); en la segunda relativa, usa el indicativo porque se compremete con el valor de verdad de su enunciado; lo cree por experiencia y por lo tanto no sería cancelable (en su mundo, lo que ha de pasar necesariamente debe ocurrir).

La ventaja de tal explicación radica en que nos permite aclarar por qué, a pesar de que la [+/-especificidad] no depende necesariamente del modo verbal, aún persiste la escogencia de uno u otro modo en las relativas. Bien podría pensarse que, si el subjuntivo se está convirtiendo en un rasgo redundante para marcar la especificidad del antecedente, entonces no debería darse la variación modal. Sin embargo, el hablante sigue haciendo la elección y esto solo podría explicarse si se analiza desde un ángulo pragmático en casos tan específicos como el anterior.

La desventaja de esta posición radica en que solamente incluiría aquellos casos de relativos de generalización que, además de su grado semántico inespecífico generalizado, tienden a expresar una acción orientada hacia el futuro, dos rasgos tradicionalmente endosados al subjuntivo.

Por otra parte, existen otros casos de relativos de generalización en los que la explicación pragmática no funciona: supongamos que ciertos niños participan en determinado juego y el que da las reglas afirma: El que llegue primero gana o El que llega primero gana. En los dos enunciados el hablante expresa la certeza de que va a existir un ganador y de que alguien llegará primero, aunque no se sepa quién. Aquí, el significado de los modos se neutraliza y, en su lugar, la semántica general del enunciado se encarga de marcar la no especificidad del antecedente. Ambos enunciados tienen el mismo significado y, por ello, subjuntivo e indicativo son, en estos entornos, alotactos en variación libre.

En la siguiente pareja de enunciados ninguna de las explicaciones anteriores es satisfactoria: El que limpia la casa es un tonto y El que limpie la casa es un tonto. Existe variación semántica entre ambas frases en relación con la especificidad del antecedente: en el primero, es [+específico]; en el segundo, [-específico].

Como se puede apreciar, el criterio de [+/-especificidad] no es determinante en la selección modal de este tipo de oraciones ya que, como se demostró, ese rasgo está marcado por otros elementos dentro del enunciado. La aparición del subjuntivo o el indicativo no conlleva variación semántica significativa en muchos de los casos $\mathrm{y}$, por consiguiente, no se puede afirmar que sea ésta definitoria del rasgo apuntado.

\footnotetext{
${ }^{16}$ Op. cit.
} 
A pesar de ello, el hablante elige una forma $u$ otra y ante esta perspectiva optamos por acudir a principios pragmáticos que dieran una luz sobre el porqué de esta selección. A pesar de que acudiendo a lo pragmático se pueden dilucidar ciertos casos (relativas de generalización), los mismos principios no son aplicables a la generalidad de los enunciados.

La otra posibilidad de explicación podría ser plantear que la especificidad del antecedente aún cumple un papel en la selección modal, pero no en términos binarios [+/-] sino como un continuum en el que, cuanto más se acerque el enunciado a la [-especificidad] mayores son las posibilidades de selección del subjuntivo sobre el indicativo y viceversa. Desde esta perspectiva, habría que plantear que la especificidad del antecedente está marcada primariamente por otros elementos que no son el modo verbal y que la elección modal resulta de una especie de atracción del mayor o menor grado de especificidad que el enunciado haya adquirido en virtud de esos otros marcadores.

De una u otra manera, debe notarse la inestabilidad del sistema verbal en esta parcela del habla culta costarricense. De hecho, se puede afirmar que el subjuntivo en las relativas no responde ya a los criterios definidos tradicionalmente y que más bien se ha llegado a una suerte de neutralización modal en estos entornos específicos.

Por ello y tomando en cuenta los casos anteriores, no podríamos esperar una explicación unitaria para el uso del subjuntivo en las frases de relativo, sino más bien proponer que existe un haz de factores que inciden en la elección modal, como creemos haber demostrado en las páginas anteriores. 